\title{
Nucleolus organizer regions in Physalaemus cuvieri (Anura, Leptodactylidae), with evidence of a unique case of Ag-NOR variability
}

\author{
ANA PAULA ZAMPIERI SILVA ${ }^{1}$, CÉLIO F. B. HADDAD ${ }^{2}$ and SANAE KASAHARA ${ }^{1}$ \\ ${ }^{i}$ Departamento de Biologia, Instituto de Biociencias, Universidade Estadual Paulista, UNESP, Rio Claro, SP, \\ Brasil \\ ${ }^{2}$ Departamento de Zoologia, Instituto de Biociências, Universidade Estadual Paulista, UNESP, Rio Claro, SP, \\ Brasil
}

\begin{abstract}
Silva, A. P. Z., Haddad, C. F. B. and Kasahara, S. 1999. Nucleolus organizer regions in Physalaemus cuvieri (Anura, Leptodactylidae), with evidence of a unique case of Ag-NOR variability._-Hereditas 131: 135-141. Lund, Sweden. ISSN 0018-0661. Received May 28, 1999. Accepted September 14, 1999
\end{abstract}

\begin{abstract}
We studied ten specimens of Physalaemus cuvieri collected at different localities in Brazil using conventional staining and banding techniques. All specimens had $2 n=22$. There were karyotypic variants: distinct patterns in the number and chromosome localization of Ag-NORs as well as in the corresponding secondary constrictions. Preliminary C-banding patterns obtained for specimens from two localities are also suggestive of karyotypic differentiation in $P$. cuvieri.

Sanae Kasahara, Departamento de Biologia, Instituto de Biociências, Universidade Estadual Paulista, UNESP, CP 199, CEP 13506-900, Rio Claro, SP, Brasil. E-mail: kasahara@rc.unesp.br
\end{abstract}

The genus Physalaemus of the subfamily Leptodactylinae comprises 39 species which are distributed throughout Mexico and South America (FROST 1985; Duellman 1993; Pombal and Madureira 1997).

To date, about 20 species (four Physalaemus were not taxonomically identified) have been karyotyped, as reported in reviews by KING (1990), KuRAMOTO (1990), and Silva (1998). The cytogenetic studies were mainly performed on conventionally stained chromosome preparations; only a few of the descriptions have been based on differential staining techniques. All species have a diploid number of 22 chromosomes. By examining the available karyograms (BEÇA 1968; BeCAK et al. 1970; Denaro 1972; De LuCCA et al. 1974; Silva 1998), it was possible to recognize two basic karyotypic formulas for the genus. One comprises only metacentrics and submetacentrics whereas the other, although very similar to the former, also includes a pair of small telocentric chromosomes.

Here, we report the results of a cytogenetic analysis on Physalaemus cuvieri collected at different localities in Brazil, using conventional Giemsa staining, AgNOR staining, and $\mathrm{C}$-banding. We observed a very unusual intraspecific variation in the number and chromosome localization of the nucleolus organizer regions.

\section{MATERIAL AND METHODS}

We studied ten males of Physalaemus cuvieri collected in the State of Rio Grande do Sul (RS) and in the State of São Paulo (SP), Brazil (Table 1): 2 specimens from Santa Maria, RS, 1 specimen from Boracéia, SP, 1 specimen from Itatiba, SP, and 6 specimens from Rio Claro, SP. The specimens included in the sample from Rio Claro, SP, were caught in two distinct regions of this municipality: 4 males in Sítio Santa Marta (site A) and 2 males in Sítio Canta Claro (site B). These sites are located about $15 \mathrm{~km}$ from each other and about 15 $\mathrm{km} \mathrm{N}$ and NW, respectively, from Rio Claro, SP. The voucher specimens were deposited in the amphibian collection of the Departamento de Zoologia, Instituto de Biociências, UNESP, Rio Claro, SP, Brazil.

Chromosome spreads were obtained from direct cytological preparations of bone marrow, liver, spleen, and testes of animals treated with colchicine, according to the procedure of BALDISSERA et al. (1993), with minor modifications.

To improve the mitotic index, we injected phytohemagglutinin-P (Difco) in some specimens before colchicine treatment, at the proportion of 0.1 $\mathrm{ml} / 10 \mathrm{~g}$ body weight, 48 to 72 hours before sacrifice. This procedure was based on BAKER et al. (1971) and WILEY (1982). Conventional staining was made with Giemsa diluted in phosphate buffer, $\mathrm{pH}$ 6.8. Ag-NOR staining was obtained by the technique of HowELL and BLACK (1980) and C-banding patterns, according to SUMNER (1972). 


\section{RESULTS}

The diploid complement of Physalaemus cuvieri is $2 \mathrm{n}=22$. It consists of metacentric and submetacentric chromosomes: the first seven pairs are large to medium-sized and the remaining four pairs are of small size. Due to variable number and chromosome localization of the nucleolus organizer regions, karyotypic variants were recognized in the sample of $P$. cuvieri.

The specimens from Santa Maria, RS, and Boracéia, SP (Table 1) had a secondary constriction at the terminal region of the short arms of chromosome pair 11 , visualized in both or in only one of the homologues (Fig. 1a). In these specimens, there was a single pair of Ag-NORs per metaphase spread, located in the secondary constriction of pair 11 (Fig. 1a).

In the specimen from Itatiba, SP (Table 1), a conspicuous secondary constriction was observed in chromosome pair 8 in the interstitial region of the long arms (Fig. 1b). The Ag-NORs were located in the secondary constriction of both homologues of pair 8 (Fig. Ib).

The frogs in the sample from Rio Claro, SP, which were collected at two distinct sites (Table 1), showed a wide range of chromosome localization for multiple nucleolus organizer regions, so that other karyotypic variants were recognized in $P$. cuvieri. In one male collected at site A, the secondary constriction and Ag-NOR stains were located interstitially in the long arms of both chromosomes of pair 8 and at the terminal region in the short arms of one of the

Table 1. Collection locality, number of specimens, and chromosome location of $\mathrm{Ag}$-NORs

\begin{tabular}{lll}
$\begin{array}{l}\text { Collection } \\
\text { Locality }\end{array}$ & $\begin{array}{l}\text { Number of } \\
\text { Specimens }\end{array}$ & $\begin{array}{l}\text { Location of the } \\
\text { Ag-NORs }\end{array}$ \\
\hline $\begin{array}{l}\text { Santa Maria, } \\
\quad 2\end{array}$ & $11 \mathrm{p}$ ter $11 \mathrm{p}$ ter \\
$\quad \begin{array}{l}\text { RS } \\
\text { Boracéia, SP }\end{array}$ & 1 & $11 \mathrm{p}$ ter $11 \mathrm{p}$ ter \\
Itatiba, SP & 1 & $8 \mathrm{q}$ int $8 \mathrm{q}$ int \\
Rio Claro, SP & 6 & $8 \mathrm{q}$ int $8 \mathrm{q}$ int $11 \mathrm{p}$ ter \\
site A & 1 & $8 \mathrm{q}$ int $8 \mathrm{q}$ int $11 \mathrm{p}$ ter \\
“ & 2 & $\mathrm{q}$ int $\mathrm{q}$ ter \\
& 1 & $8 \mathrm{q}$ int $8 \mathrm{q}$ int $3 \mathrm{q}$ int \\
“ & 1 & $8 \mathrm{q}$ int $8 \mathrm{q}$ int $11 \mathrm{p}$ ter \\
site B & $1^{*}$ & $5 \mathrm{p}$ int $7 \mathrm{q}$ int $8 \mathrm{q}$ int \\
“ & $1^{*}$ & $\mathrm{p}$ int $8 \mathrm{q}$ int $11 \mathrm{p}$ ter
\end{tabular}

$\mathrm{p}=$ short $\mathrm{arm} ; \mathrm{q}=$ long $\mathrm{arm} ;$ int $=$ interstitial; ter $=$ terminal.

* specimens with intraindividual variation in Ag-NOR patterns. The maximum number of Ag-NORs is indicated for each specimen.

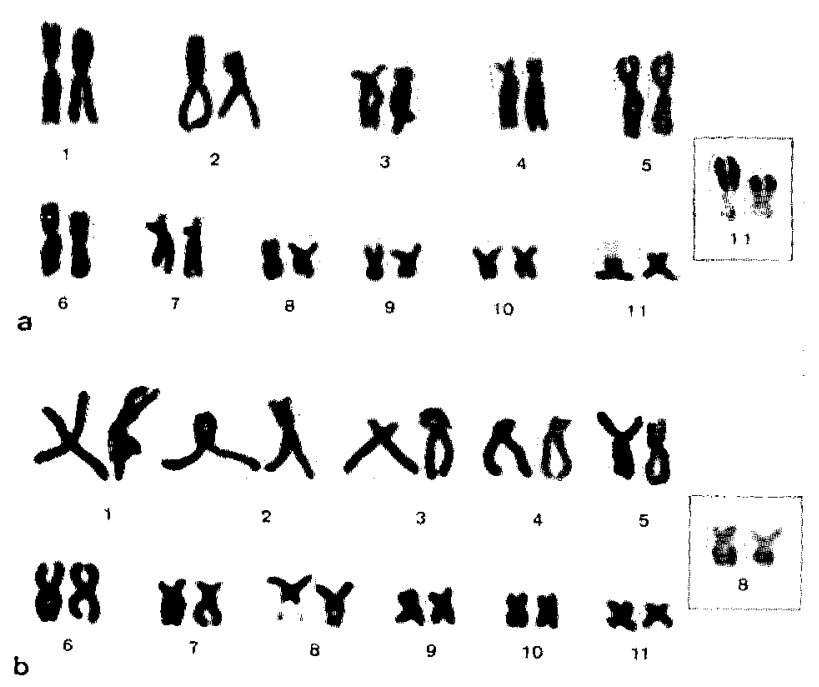

Fig. 1. a and b. Giemsa-stained karyotypes and Ag-NORstained chromosomes (inset) of Physalaemus cuvieri $(2 \mathrm{n}=$ 22). a Male from Santa Maria, RS, presenting secondary constriction and Ag-NOR in the chromosomes of pair 11. b Male from Itatiba, SP, presenting secondary constriction and Ag-NOR in the chromosomes of pair 8.

homologues of pair 11 (Fig. 2a). One specimen collected at site B presented the same karyotype variant, but secondary constrictions were observed only in one of the chromosomes 8 and in one chromosome 11. The majority of its Ag-stained metaphase spreads exhibited three NORs. Some rare metaphases showed two Ag-NORs: one in chromosome 8 and one in chromosome 11.

Two other frogs from Rio Claro, SP, collected at the site A (Table 1) also had nucleolus organizer regions in pair 8 , in the interstitial region of the long arms, and in one of the chromosomes of pair 11 (Fig. $2 b)$. The secondary constriction was not always seen in both homologues of pair 8. In this karyotypic variant, one chromosome 11 showed altered morphology, i.e., it was subtelocentric, with a terminal secondary constriction in the short arms as well as one or two interstitial secondary constriction in the long arms (Fig. 2b; Fig. 4b). Ag-NOR staining was obtained for only one of the specimens. It showed Ag-NORs in both chromosomes 8 and an atypical pattern along chromosome 11: one $\mathrm{Ag}-\mathrm{NOR}$ was located at the terminal position of the short arms, one at the terminal position of the long arms, and one in the interstitial region of the long arms (Fig. $2 b$ ). Even though in this specimen one of the homologues of pair 5 sporadically had a secondary constriction in the short arms (Fig. 2b), Ag-NOR staining was never observed in any of the elements of this chromosome pair.

Another frog collected at site A (Table 1) had interstitial secondary constrictions in the long arms of 
the two chromosomes of pair 8 and in one of the homologues of pair 3 (Fig. 2c). These secondary constrictions correspond to the Ag-NORs in the chromosome complement of this specimen (Fig. 2c).

Finally, one male collected at site B (Table 1) had multiple nucleolus organizer regions scattered along a larger number of chromosomes. Fig. 3 shows the maximum number of Ag-NORs, distributed on five different chromosomes, but the most frequent pattern consisted of Ag-NORs located in four chromosomes: in the interstitial region of the short arms of one chromosome 5 , in the interstitial region of the long arms of one chromosome 7 , in the interstitial region in both of the short arms and of the long arms of one chromosome 8 , and at the terminal region of the short arms of one chromosome 11. Other less frequent patterns were observed in the cell sample, in which some nucleolus organizer regions did not appear as transcriptionally active Ag-NOR stains. The conventionally stained metaphase spreads in this

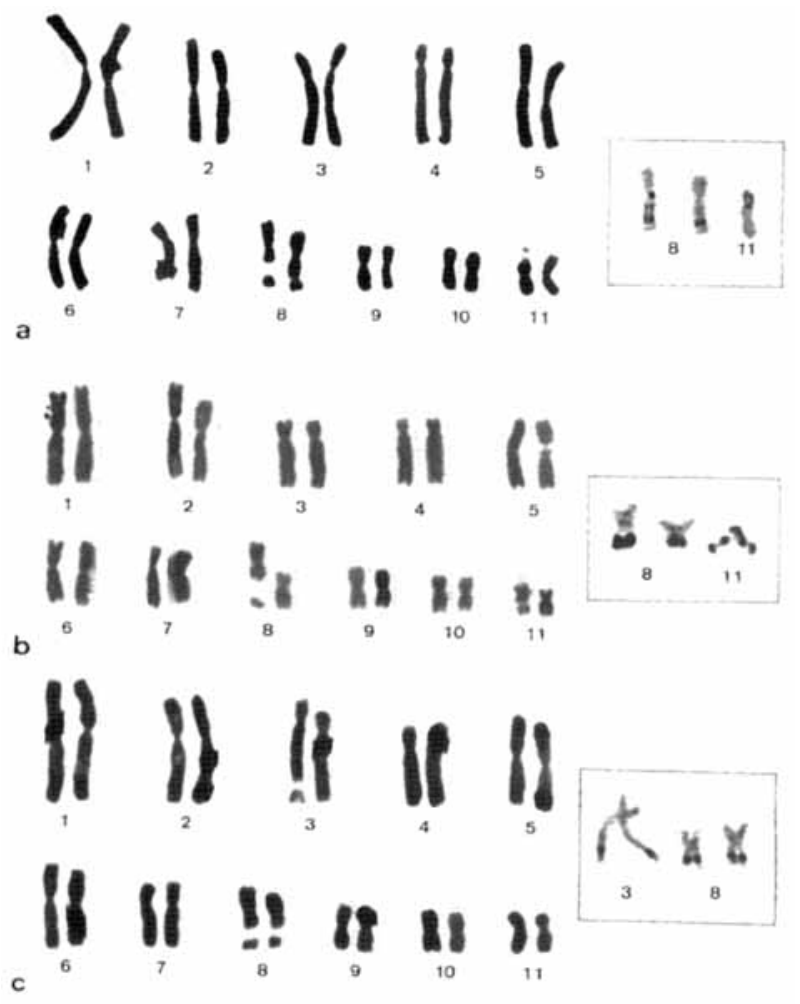

Fig. 2. a-c. Giemsa-stained karyotypes and Ag-NORstained chromosomes (inset) of Physalaenus cuvieri $(2 \mathrm{n}=$ 22) from Rio Claro, SP (site A). a Male presenting secondary constriction and Ag-NOR in the chromosomes of pair 8 and in one chromosome 11. b Male presenting secondary constriction and Ag-NOR in the chromosomes of pair 8 and in one chromosome 11 with altered morphology. c Male presenting secondary constriction and AgNOR in the chromosomes of pair 8 and in one chromosome 3 . specimen rarely had negative heteropycnotic secondary constrictions. This structure appeared in general in the interstitial region of the long arms of one chromosome 8.

At least $8 \mathrm{Ag}$-stained metaphase spreads were analyzed for each specimen. Except for those with intraindividual Ag-NOR variation, the remaining specimens of $P$. cuvier $i$ had an identical pattern in all metaphase spreads from each sample.

Among the specimens we frequently observed conspicuous size differences between homologous AgNORs (Fig. 1a; Fig. 2a). This finding could be attributed to differential genetic activity of rDNA segments. In some cases, however, the heteromorphism was certainly due to a duplication of rDNA content as evidenced by tandem duplicated Ag-NOR blocks (Fig. 2a).

Chromosome preparations of two specimens from distinct localities were studied with the C-banding technique (Fig. 4a; Fig. 4b). The specimen from Santa Maria, RS, showed a small amount of constitutive heterochromatin which appeared in general as faintly stained C-bands in some chromosomes (Fig. 4a). The chromosomes of pair 9 had an additional interstital band at the proximal region of the long arms. A prominent C-band was observed at the distal end of the long arms in one of the homologues of pair 3. One male from Rio Claro, SP, collected at site $\mathrm{A}$, also showed $\mathrm{C}$-bands in the centromeric region of some chromosomes (Fig. 4b). A conspicuous telomeric C-band was observed in the long arms of one of the homologues of pair 2. Furthermore, interstitial $\mathrm{C}$-bands were seen in the short arms of pair 2 and pair 5 as well as in the long arms of pair 9 .

At meiosis these frogs had 11 bivalents in diplotene and metaphase I cells and 11 chromosomes in metaphase II cells. The bivalents were usually ringshaped due to terminal chiasmata in both arms. Occasionally, we observed a rod-shaped bivalent due to the presence of only one terminal chiasma.

\section{DISCUSSION}

The $2 \mathrm{n}=22$ chromosome complement of Physalaemus cuvieri that we studied had exclusively metacentrics and submetacentrics. This agrees with that previously described by BECAK et al. (1970) for specimens collected in the State of Bahia, Brazil. This karyotypic formula is consistent for all species of Physalaemus included in the cuvieri morphological group, like $P$. aguirrei, $P$. albifrons, $P$. centralis, $P$. cicada. $P$. gracilis, $P$. kroyeri, and $P$. soaresi, which have been karyotyped up to now (DENARO 1972; DE LuCCA et al. 1974; OlIVEIRA and YONENAGA-YASSUDA 1995). 


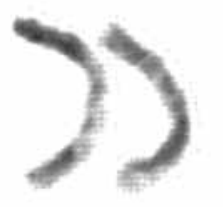

1

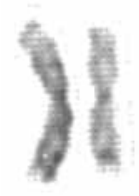

6

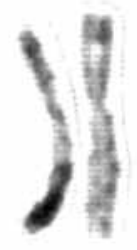

2

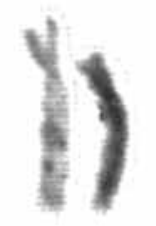

3

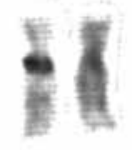

7

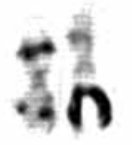

8

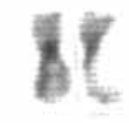

9

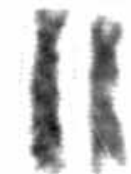

4

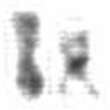

10

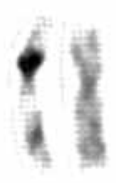

5

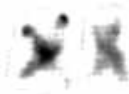

11

Fig. 3. Ag-NOR stained karyotype of Physalaemus cuvieri $(2 \mathrm{n}=22)$ from Rio Claro, SP (site B) presenting Ag-NORs in one chromosome 5 , one chromosome 7 , two chromosomes 8 , and one chromosome 11 .

It has been shown that a general feature in anurans is the presence of a single pair of Ag-NORs, almost always conservatively localized in homoeologous chromosome pairs in species belonging to the same or to closely related species groups (SCHMID 1982; KING 1990; SCHMID et al. 1990). Contrary to this finding, the analysis of ten specimens of $P$. cuvieri from different localities showed a very unusual intraspecific variation in the Ag-stained nucleolus organizer regions.

The karyotypic variants in P. cuvieri are related either to a shift in the position of a single pair of Ag-NORs or to the occurrence of multiple Ag-NORs in variable chromosome positions. Ag-NORs were located in chromosomes 11 for the specimens from Santa Maria, RS, and Boracéia, SP, or in chromosomes 8 for the male from Itatiba, SP. Although found in only one specimen, the Ag-NOR at this position is not fortuitous in that the whole sample of $P$. cuvieri from Rio Claro, SP, also had pair 8 as a major pair of Ag-NORs bearing chromosomes. Surprisingly, all six specimens showed at least one additional chromosome bearing Ag-NORs, so that four other distinct patterns of active Ag-NORs were recognized in P. cuvieri. The additional Ag-NORs always appear in heteromorphic condition in pair 3,5, 7 , or 11. The homologous elements in each chromosome pair had no vestige of Ag-NOR staining or secondary constriction. Nevertheless, we could not rule out the hypothesis that these chromosomes carry, in fact, latent nucleolus organizer regions, because Ag technique only stains rDNA segments that were genetically active in the preceding interphase.

Intraindividual variability in the active Ag-NOR pattern was also observed in $P$. cuvieri. Considering that the nucleolus organizer regions are mendelian inherited characteristics, a differential regulation in the expression of ribosomal genes may be operating in distinct cell lines within the same individual.

Based on the most frequent position of the secondary constriction in species belonging to Physalaemus cuvieri species group, we might suppose that the standard localization of the nucleolus organizer regions in $P$. cuvieri is that of pair 11. In $P$. centralis and $P$. soaresi secondary constriction was reported in the smallest pair of the complement (DENARO 1972; DE LUCCA et al. 1974). Physalaemus albifrons was described by DENARO (1972) as carrying a secondary constriction in pair 11 and in pair 2 but OLIVEIRA and YoNENAGA-YASSUdA (1995) reported secondary constriction in pair 9 for specimens from another locality. For the remaining species of the $P$. cuvieri group there is no reference to the presence of this chromosome structure. Although more consistent data are still necessary to determine which is the standard Ag-NOR pattern in $P$. cuvieri, whether in pair 11 or in pair 8 , the multiple Ag-NORs observed in the sample from Rio Claro, SP, may be regarded as a derived condition.

The chromosomes of pair 8 had, in general, the same pattern of $\mathrm{Ag}-\mathrm{NOR}$ staining, always in the interstitial region of the long arms. However, in one male, one chromosome 8 presented an additional Ag-NOR in the short arms, but no visible change in chromosome morphology was observed. Chromosome 11 also exhibited a discrepant Ag-NOR pattern in one male, carrying three Ag-NOR stains along its length. This chromosome 11 was probably rearranged as it had a subtelocentric morphology as well as peculiar patterns of secondary constrictions and AgNOR staining. At present, we have no means to determine the exact mechanism responsible for the 
repatterning of this chromosome 11, but multiple structural rearrangements, like minute insertions and/ or small inversions, as pointed out by KING (1980) in the genus Litoria, seems to be involved.

Intraspecific variation in Ag-NOR patterns has been reported in some anurans such as Hyla chrysoscelis and H. versicolor (WILEY et al. 1989), Bufo terrestris (FOOTE et al. 1991), Rana japonica (MIUra 1994), Agalychnis callidryas (SCHMID et al. 1995), and Hyla ebraccata (KAISER et al, 1996). In these species, distinct chromosome sites as well as variations in the number of nucleolus organizer regions, visualized by Ag-staining or in situ hybridization techniques, were described in karyotypes from different populations. Furthermore, extra Ag-NORs on the same chromosome were observed in the species $A$. callidryas (SCHMID et al. 1995), as also reported for chromosomes 8 and 11 of some specimens of $P$. cuvieri in the present sample. Some possible mechanisms have been suggested by FoOTE et al. (1991) and SCHMID et al. (1995) to explain the origin of the intraspecific variations in number and chromosome locations of nucleolus organizer regions, which we summarize as follows: a. occurrence of reciprocal translocations and/or inversions; $b$. transposition of rDNA segments by means of mobile genetic elements; c. amplification of single "orphan-like" rDNA genes, forming functional and identifiable nucleolus organizer regions; d. reinsertion errors during the extrachromosomal amplification of rDNA in the course of oogenesis.

Conventional types of chromosomal rearrangements seem to be a less probable explanation for the karyotypic variants in $P$. cuvieri, since in this case changes in the chromosome arm ratio would be expected in the karyotype. Except for the alteration in the morphology of chromosome 11 in one case, no other evidence of chromosome repatterning was ob-

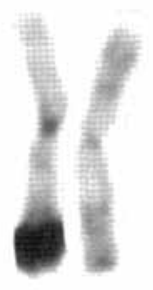

1

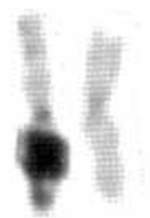

6

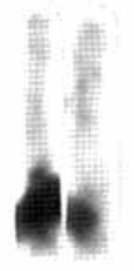

2

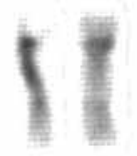

7

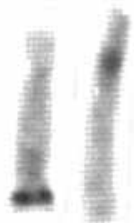

3

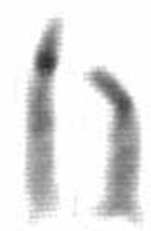

4

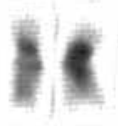

9

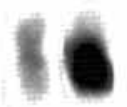

10

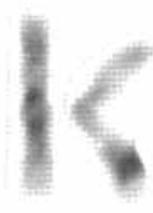

5

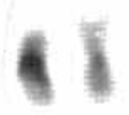

11

a

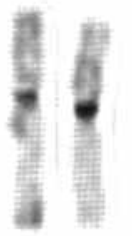

1

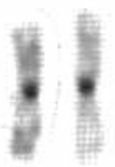

6

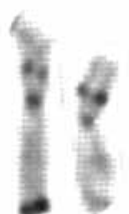

2

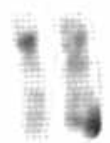

7
8

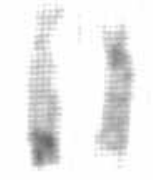

3

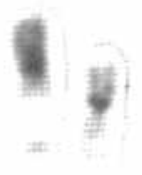

8
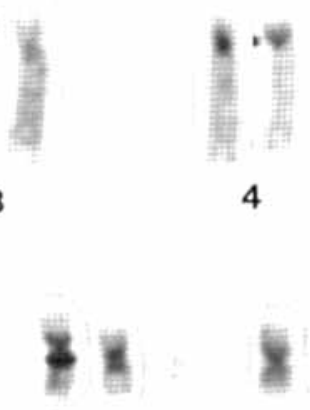

9
4

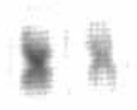

10

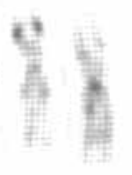

5

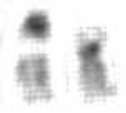

11

b

Fig. 4. a and b. C-banded karyotypes of Physalaemus cuvieri $(2 \mathrm{n}=22)$. a Male from Santa Maria, RS. b Male from Rio Claro, SP (site A). 
served. For this reason, the hypothesis based on the remaining events listed above seems to be more consistent with the observed data. Amplification of single ribosomal copies, excision of rDNA segments from one chromosome site and reinsertion at another site might explain the shift in number and localization of the nucleolus organizer regions in the chromosome complement of $P$. cuvieri, but all of these possibilities should still be tested.

Physalaemus cuvieri is widely distributed in Brazil, throughout the Northeastern, Central, and Southeastern regions (FROST 1985). These frogs are typically found in open areas. Although specimens were collected at a limited number of localities and the sample size from each collecting sites was quite small, there are strong indications that inter- and intrapopulational variations in Ag-NOR patterns occur in this species. Moreover, the secondary constrictions in the short arms of chromosome pairs 2 and 3 in the specimens from the State of Bahia (Beçak et al. 1970) could be indicative of a further karyotypic variant occurring in P. cuvieri. Additional cytogenetic data for a larger number of specimens from new collection sites as well as from previously visited localities are required for a better characterization of $\mathrm{Ag}-\mathrm{NOR}$ variability in $P$. cuvieri. It would be interesting, for example, to verify if the specimens collected at site $A$ and site B from Rio Claro, SP, have more than one karyotypic variant in common.

Although very scanty, preliminary C-banding data available for specimens from two different localities indicate that their karyotypes also differ regarding the distribution of some interstitial and telomeric bands. Further $\mathrm{C}$-banding analyses in specimens from distinct localities as well as bearing variable Ag-NOR patterns will be of importance to evaluate the extent of the karyotypic differentiation occurring in $P$. $c u$ vieri. Data on external morphological traits or on vocalization might be also important to complement these studies.

\section{ACKNOWLEDGEMENTS}

We are grateful to Dr. Alejo Mesa and Paulo C. A. Garcia for collecting some specimens of $P$. cuvieri. We also thank Rogilene Aparecida Prado and Carmem Silvia Mengardo for technical assistance. This work was supported by $\mathrm{CNPq}$ and FAPESP.

\section{REFERENCES}

Baker RJ, Bull JJ and Mengden GA, (1971). Chromosomes of Elaphe subocularis (Reptilia: Serpentes), with the description of an in vivo technique for preparation of snake chromosome. Experientia 27: 1228-1229.
Baldissera FA Jr, Oliveira PSL and Kasahara S, (1993). Cytogenetics of four Brazilian Hyla species (AmphibiaAnura) and description of a case with a supernumerary chromosome. Rev. Brasil. Genet. 16: 335-345.

Beçak ML, (1968). Chromosomal analysis of eighteen species of anura. Caryologia 21: 191-208.

Beçak ML, Denaro L and Beçak W, (1970). Polyploidy and mechanisms of karyotypic diversification in Amphibia. Cytogenetics 9: 225-238.

De Lucca EJ, Jim J and Foresti F, (1974). Chromosomal studies in twelve species of Leptodactylidae and one Brachycephalidae. Caryologia 27: 183-192.

Denaro L, (1972). Karyotypes of Leptodactylidae anurans. J. Herpetol. 6: 71-74.

Duellman WE, (1993). Amphibian Species of the World: Additions and Corrections. University of Kansas Printing Service, Lawrence.

Foote DL, Wiley JE, Little ML and Meyne J, (1991). Ribosomal RNA gene site polymorphism in Bufo terrestris. Cytogenet. Cell Genet. 57: 196-197.

Frost DR, (1985). Amphibian Species of the World. A Taxonomic and Geographical Reference. Allen Press and Association of Systematics Collections, Lawrence.

Howell WM and Black DA, (1980). Controlled silver-staining of nucleolus organizer regions with a protective colloidal developer: 1-step method. Experientia 36: 1014-1015.

Kaiser H, Mais C, Bolaños F and Steinlein C, (1996). Chromosomal investigation of three Costa Rican frogs from the 30-chromosome radiation of Hyla with description of a unique geographic variation in nucleolus organizer regions. Genetica 98: 95-102.

King M, (1980). C-banding studies on Australian hylid frogs: secondary constriction structure and the concept of euchromatin transformation. Chromosoma 80: 191217.

King M, (1990). Amphibia. In: Animal Cytogenetics. Amphibia, 4. Chordata 2. (ed B John), Gebruder Borntraeger, Berlin.

Kuramoto M, (1990). A list of chromosome numbers of anuran amphibiams. Bull. Fukuoka Univ. Educ. 39: $83-127$.

Miura I, (1994). Sex chromosome differentiation in the Japanese Brown frog, Rana japonica. II. Sex-linkage analyses of the nucleolar organizer regions in chromosomes no. 4 of the Hiroshima and Saeki populations. Zool. Sci. 11: $807-815$.

Oliveira RP, Yonenaga-Yassuda Y, (1995). Descrição cariotípica de algumas espécies de anfibios brasileiros. Brazil. J. Genet. 18: 474 (Suppl.).

Pombal JP Jr and Madureira CA, (1997). A new species of Physalaemus (Anura, Leptodactylidae) from the Atlantic rain forest of Northeastern Brazil. Alytes 15: $105-112$.

Schmid M, (1982). Chromosome banding in Amphibia. VII. Analysis of structure and variability of NORs in Anura. Chromosoma 87: 327-344.

Schmid M, Steinlein C, Nanda I and Epplen JT, (1990). Chromosome banding in Amphibia. In: Cytogenetics of Amphibians and Reptiles (ed E Olmo), Birkhauser Verlag, Basel, p.21-45.

Schmid M, Feichtinger W, Weimer R, Mais C, Bolaños F and León P, (1995). Chromosome banding in Amphibia. XXI. Inversion polymorphism and multiple nucleolus 
organizer regions in Agalychnis callidryas (Anura, Hylidae). Cytogenet. Cell Genet. 69: 18-26.

Silva APZ, (1998). Estudos citogenéticos de anuros da Família Leptodactylidae (Amphibia), com técnicas de coloração diferencial. M.S. Thesis, Universidade Estadual Paulista, Rio Claro, São Paulo. Brasil.

Sumner AT, (1972). A simple technique for demonstrating centromeric heterochromatin. Exp. Cell Res. 75: 304-306.
Wiley JE, (1982). Chromosome banding patterns of tree frogs (Hylidae) of the Eastern United States. Herpetologica 38: $507-520$.

Wiley JE, Little ML, Romano MA, Blount DA and Cline GR, (1989). Polymorphism in the location of the $18 \mathrm{~S}$ and $28 \mathrm{~S}$ rRNA genes on the chromosomes of the diploidtetraploid tree frogs Hyla chrysoscelis and $\mathrm{H}$. versicolor. Chromosoma 97: 481-487. 